\title{
Genome-wide analysis in UK Biobank identifies four loci associated with mood instability and genetic correlation with major depressive disorder, anxiety disorder and schizophrenia
}

Joey Ward ${ }^{1}$, Rona J. Strawbridge (10 ${ }^{1,2}$, Mark E. S. Bailey ${ }^{3}$, Nicholas Graham¹, Amy Ferguson ${ }^{1}$, Donald M. Lyall ${ }^{1}$, Breda Cullen', Laura M. Pidgeon', Jonathan Cavanagh', Daniel F. Mackay', Jill P. Pell', Michael O'Donovan (1) ${ }^{4}$, Valentina Escott-Price ${ }^{4}$ and Daniel J. Smith (10 ${ }^{1}$

\begin{abstract}
Mood instability is a core clinical feature of affective and psychotic disorders. In keeping with the Research Domain Criteria approach, it may be a useful construct for identifying biology that cuts across psychiatric categories. We aimed to investigate the biological validity of a simple measure of mood instability and evaluate its genetic relationship with several psychiatric disorders, including major depressive disorder (MDD), bipolar disorder (BD), schizophrenia, attention deficit hyperactivity disorder (ADHD), anxiety disorder and post-traumatic stress disorder (PTSD). We conducted a genome-wide association study (GWAS) of mood instability in 53,525 cases and 60,443 controls from UK Biobank, identifying four independently associated loci (on chromosomes 8, 9, 14 and 18), and a common single-nucleotide polymorphism (SNP)-based heritability estimate of $\sim 8 \%$. We found a strong genetic correlation between mood instability and $\operatorname{MDD}\left(r_{\mathrm{g}}=0.60, \mathrm{SE}=0.07, p=8.95 \times 10^{-17}\right)$ and a small but significant genetic correlation with both schizophrenia $\left(r_{\mathrm{g}}=0.11, \mathrm{SE}=0.04, p=0.01\right)$ and anxiety disorders $\left(r_{\mathrm{g}}=0.28, \mathrm{SE}=0.14, p=0.04\right)$, although no genetic correlation with BD, ADHD or PTSD was observed. Several genes at the associated loci may have a role in mood instability, including the DCC netrin 1 receptor (DCC) gene, eukaryotic translation initiation factor 2B subunit beta (elF2B2), placental growth factor (PGF) and protein tyrosine phosphatase, receptor type D (PTPRD). Strengths of this study include the very large sample size, but our measure of mood instability may be limited by the use of a single question. Overall, this work suggests a polygenic basis for mood instability. This simple measure can be obtained in very large samples; our findings suggest that doing so may offer the opportunity to illuminate the fundamental biology of mood regulation.
\end{abstract}

\section{Introduction}

Mood instability is a common clinical feature of affective and psychotic disorders, particularly major depressive disorder (MDD), bipolar disorder (BD) and

\footnotetext{
Correspondence: Daniel J Smith (daniel.smith@glasgow.ac.uk) ${ }^{1}$ Institute of Health and Wellbeing, University of Glasgow, Glasgow, UK 2Department of Medicine Solna, Karolinska Institute, Stockholm, Sweden Full list of author information is available at the end of the article
}

schizophrenia ${ }^{1}$. It may also be relatively common in the general population, estimated to affect $\sim 13 \%$ of individuals $^{2}$. As a dimensional psychopathological trait, it is potentially a useful construct in line with the Research Domain Criteria approach ${ }^{3}$. Mood instability may be of fundamental importance for understanding the pathophysiology of MDD and BD, as well as conditions such as borderline personality disorder, anxiety disorders, 
attention deficit hyperactivity disorder (ADHD) and psychosis $^{4}$. This trait is reported by $40-60 \%$ of individuals with $\mathrm{MDD}^{5}$ and is recognised as part of the prodromal stage of $\mathrm{BD}^{6}$. In established $\mathrm{BD}$, it is a clinical feature that independently predicts poor functional outcome ${ }^{7}$. Furthermore, general population twin studies suggest that additive genetic effects account for $40 \%$ of the variance in measures of affect intensity and $25 \%$ of the variance in affective liability ${ }^{8}$.

Population-based studies such as the Adult Psychiatric Morbidity Survey (APMS) have defined mood instability based on responses to a single question, while clinical studies have made use of more detailed rating scales ${ }^{4}$. However, there is a lack of consensus about how best to measure and classify mood instability, and none of the currently available instruments adequately capture intensity, speed and frequency of affective change, or physiological and behavioural correlates. A recent systematic review proposed that mood instability be defined as 'rapid oscillations of intense affect, with a difficulty in regulating these oscillations or their behavioural consequences' $^{\prime 9}$. Applying this definition will require the future development and validation of a multidimensional assessment of mood instability, which is currently not available.

Within the UK Biobank population cohort of over 0.5 million individuals ${ }^{10}$, the baseline assessment interview contained a question of relevance to mood instability, specifically: 'Does your mood often goes up and down?' This is similar to the question for mood instability used within the APMS ('Do you have a lot of sudden mood changes, suffered over the last several years'). Hypothesising that this simple question taps into pathological mood instability, we predicted that it would be more commonly endorsed by individuals within UK Biobank with MDD and BD, compared to individuals with no psychiatric disorder. Moreover, under the hypothesis that this trait has cross-disorder pathophysiological relevance, we predicted that a genome-wide association study (GWAS) might identify shared genetic liability to mood instability and risk for psychiatric disorders in which disordered mood is a feature, including MDD, BD, schizophrenia, ADHD, anxiety disorder and post-traumatic stress disorder (PTSD). Given the size of the sample, we also aimed to identify loci associated with this measure of mood instability.

\section{Materials and methods \\ Sample}

UK Biobank is a large cohort of more than 502,000 United Kingdom residents, aged between 40 and 69 years ${ }^{10}$. The aim of UK Biobank is to study the genetic, environmental and lifestyle factors that cause or prevent disease in middle and older age. Baseline assessments occurred over a 4-year period, from 2006 to 2010, across 22 United Kingdom (UK) centres. These assessments were comprehensive and included social, cognitive, lifestyle and physical health measures. For the present study, we used the first genetic data release based on approximately one-third of UK Biobank participants. Aiming to maximise homogeneity, we restricted the sample to those who reported being of white UK ancestry (around 95\% of the sample).

UK Biobank obtained informed consent from all participants, and this study was conducted under generic approval from the NHS National Research Ethics Service (approval letter dated 13 May 2016, Ref 16/NW/0274) and under UK Biobank approvals for application \#6553 'Genome-wide association studies of mental health' (PI Daniel Smith).

\section{Mood instability phenotype}

As part of the baseline assessment, UK Biobank participants completed the 12 items of the neuroticism scale from the Eysenck Personality Questionnaire-Revised Short Form (EPQ-R-S) ${ }^{11}$. One of these items assesses mood instability, namely 'Does your mood often goes up and down?' Participants responding 'yes' to this question were considered to be cases of mood instability and those responding 'no' were considered controls. From the control sample, we excluded those who reported being on psychotropic medication, and those who reported a physician diagnosis of psychiatric disorder (including MDD, $\mathrm{BD}$, anxiety/panic attacks, 'nervous breakdown', schizophrenia and deliberate self-harm/suicide attempt).

After quality-control steps (detailed below) and exclusions (3679 participants responded 'don't know' and 211 responded 'prefer not to say'), the final sample for genetic analysis comprised 53,525 cases of mood instability and 60,443 controls. Mood instability cases were younger than controls (mean age 55.8 years $(\mathrm{SD}=8.05)$ vs. 57.7 years $(\mathrm{SD}=7.74) ; p<0.0001)$ and had a greater proportion of females $(55.5 \%$ vs. $49.6 \%$; $p<0.0001)$.

\section{Genotyping and imputation}

In June 2015, UK Biobank released the first set of genotypic data for 152,729 UK Biobank participants. Approximately $67 \%$ of this sample was genotyped using the Affymetrix UK Biobank Axiom array (Santa Clara, CA, USA) and the remaining 33\% were genotyped using the Affymetrix UK BiLEVE Axiom array. These arrays have over $95 \%$ content in common. Only autosomal data were available under the data release. Data were preimputed by UK Biobank as fully described in the UK Biobank interim release documentation ${ }^{12}$. Briefly, after removing genotyped single-nucleotide polymorphisms (SNPs) that were outliers, or were multiallelic or of low frequency (minor allele frequency $(\mathrm{MAF})<1 \%$ ), phasing 
was performed using a modified version of SHAPEIT2 and imputation was carried out using IMPUTE2 algorithms, as implemented in a $\mathrm{C}++$ platform for computational efficiency ${ }^{13}, 14$. Imputation was based upon a merged reference panel of $87,696,888$ biallelic variants on 12,570 haplotypes constituted from the 1000 Genomes Phase 3 and UK10K haplotype panels ${ }^{15}$. Variants with MAF $<0.001 \%$ were excluded from the imputed marker set. Stringent quality control before release was applied by the Wellcome Trust Centre for Human Genetics, as described in UK Biobank documentation ${ }^{16}$.

\section{Statistical analyses}

\section{Quality control and association analyses}

Before all analyses, further quality-control measures were applied. Individuals were removed based on UK Biobank genomic analysis exclusions (Biobank Data Dictionary item \#22010), relatedness (\#22012: genetic relatedness factor; a random member of each set of individuals with KING-estimated kinship coefficient $>0.0442$ was removed), gender mismatch (\#22001: genetic sex), ancestry (\#22006: ethnic grouping; principal component (PC) analysis identified probable Caucasians within those individuals who were self-identified as British and other individuals were removed from the analysis), and qualitycontrol failure in the UK BiLEVE study (\#22050: UK BiLEVE Affymetrix quality control for samples and \#22051: UK BiLEVE genotype quality control for samples). A sample of 113,968 individuals remained for further analyses. Of these, 53,525 were classed as cases and 60,443 were classified as controls. Genotype data were further filtered by removal of SNPs with Hardy-Weinberg equilibrium $P<10^{-6}$, with MAF $<0.01$, with imputation quality score $<0.4$ and with data on $<90 \%$ of the sample after excluding genotype calls made with $<90 \%$ posterior probability, after which 8,797,848 variants were retained.

Association analysis was conducted in PLINK ${ }^{17}$ using logistic regression under a model of additive allelic effects with sex, age, genotyping array and the first eight PCs (Biobank Data Dictionary items \#22009.01 to \#22009.08) as covariates. Sex and age were included as covariates because cases and controls differed significantly on these measures. Genetic PCs were included to control for hidden population structure within the sample, and the first eight PCs, out of 15 available in the Biobank, were selected after visual inspection of each pair of PCs, taking forward only those that resulted in multiple clusters of individuals after excluding individuals self-reporting as being of non-white British ancestry (Biobank Data Dictionary item \#22006). Overall, population structure had little impact on mood instability status. The threshold for genome-wide significance was $p<5.0 \times 10^{-8}$.
Heritability and genetic correlation between mood instability and psychiatric phenotypes

We applied Linkage Disequilibrium Score Regression (LDSR $^{18}$ to the GWAS summary statistics to estimate SNP heritability $\left(\mathrm{h}_{\mathrm{SNP}}^{2}\right)$. Genetic correlations between mood instability and MDD, BD, schizophrenia, ADHD, anxiety disorder and PTSD were also evaluated using LDSR $^{19}$ (with unconstrained intercept), a process that corrects for potential sample overlap without relying on the availability of individual genotypes ${ }^{18}$. For the MDD, BD, schizophrenia, ADHD, anxiety disorder and PTSD phenotypes, we used GWAS summary statistics provided by the Psychiatric Genomics Consortium (http://www. med.unc.edu/pgc//) ${ }^{20-25}$. Note that for the purposes of these genetic correlation analyses we re-ran the GWAS of mood instability excluding from the cases those 9865 participants who reported being on psychotropic medication, or who self-reported psychiatric disorder (MDD, $\mathrm{BD}$, anxiety/panic attacks, 'nervous breakdown', schizophrenia and deliberate self-harm/suicide attempt). This secondary GWAS output (rather than the primary GWAS reported below) was used for the genetic correlation calculations and for polygenic risk score (PRS) analyses, the rationale being that this was a more conservative approach that would avoid genetic correlations between mood instability and MDD/BD/schizophrenia/ADHD/ anxiety disorders/PTSD being driven by a subset of individuals with psychiatric disorder.

\section{PRS analysis of MDD, BD and schizophrenia as predictors of mood instability}

PRSs were created using the output of the PCG MDD 29 of 32 cohort GWAS (supplied by the MDD working group of the PGC, http://www.med.unc.edu/pgc/pgcworkgroups), BD GWAS ${ }^{20}$ and schizophrenia GWAS ${ }^{21}$. Five PRS were created for each psychiatric phenotype using $p$ value cutoffs of $p<5 \times 10^{-8}, p<0.01, p<0.05, p$ $<0.1$ and $p<0.5$, with the exception of MDD for which there were no genome-wide significant SNPs. Ambiguous SNPs, indels (insertion/deletion mutations) and SNPs with an imputation quality score of less than 0.8 were removed. LD clumping was performed via PLINK on a random sample of 10,000 individuals using an $r^{2}>0.05$ in a $250 \mathrm{~kb}$ window. SNPs were clumped into sets and filtered, selecting the SNP with the lowest $p$ value from each set. In the event that two or more SNPs from a set had the same $p$ value, the SNP with the largest beta coefficient was used. PLINK was also used to calculate the PRS to produce a per-allele weighted score with no mean imputation.

\section{PRS modelling}

Only those subjects who were used for the genetic correlation analyses were used in the PRS analyses (that is, 
PRS analyses also excluded from both case and control groups those individuals in UK Biobank with psychiatric disorder). Modelling was performed in $\mathrm{R}$ (version 3.1.2) using the glm function. Full sample and age-stratified analysis models were adjusted for age, sex, chip and PGCs $1-8$, whereas sex-stratified analysis was not adjusted for sex. Scores were then split into deciles using the ntile function of the dplyr package. Model Nagelkerke $r^{2}$ was calculated using the fmsb package.

\section{Results}

\section{Mood instability in MDD and BD within UK Biobank}

In previous work we have identified individuals within UK Biobank with a probable diagnosis of mood disorder, including cases of MDD (subdivided into single-episode MDD, recurrent moderate MDD and recurrent severe MDD) and BD, as well as non-mood disordered con$\operatorname{trols}^{26}$. These classifications were independent of response to the mood instability question or other questions from the EPQ-R-S. For the group of participants who could be classified in this way, we assessed the proportion with mood instability within each mood disorder category. All mood disorder groups had a significantly greater proportion of individuals with mood instability compared with the control group (Table 1), in which the prevalence was $35.3 \%$. This proportion was highest in the BD group (74.0\%) followed by the three MDD groups (71.7\% for recurrent severe MDD, 64.2\% for recurrent moderate MDD and $43.7 \%$ for single-episode MDD). There were too few UK Biobank participants with a reliable classification of schizophrenia, ADHD, anxiety disorder or PTSD to allow for an assessment of the prevalence of mood instability in these groups.

\section{GWAS of mood instability}

The mood instability GWAS results are summarised in Fig. 1 (Manhattan plot), Fig. 2 (QQ plot) and Table 2

Table 1 Proportion of individuals with mood instability within mood disorder groups, compared to non-mood disordered controls

\begin{tabular}{llcc}
\hline & $\begin{array}{l}\text { Mood instability } \\
\boldsymbol{N}(\%)\end{array}$ & Pearson $\boldsymbol{X}^{2}$ & $\boldsymbol{P}$ value \\
\hline BD & $1180(74.0)$ & $1.0 \times 10^{3}$ & $<0.001$ \\
Recurrent MDD, severe & $6303(71.7)$ & $4.5 \times 10^{3}$ & $<0.001$ \\
Recurrent MDD, & $9509(64.2)$ & $4.4 \times 10^{3}$ & $<0.001$ \\
moderate & & & \\
Single-episode MDD & $3403(43.7)$ & 221.1 & $<0.001$ \\
Non-mood disordered & $30,844(35.3)$ & - & - \\
controls & & & \\
\hline
\end{tabular}

$B D$ bipolar disorder, $M D D$ major depressive disorder (genome-wide significant loci associated with mood instability). Regional plots are provided in Figs. 3a-d.

Overall, the GWAS data showed modest deviation in the test statistics compared with the null $\left(\lambda_{\mathrm{GC}}=1.13\right)$; this was negligible in the context of sample size $\left(\lambda_{\mathrm{GC}} 1000=\right.$ 1.002). LDSR suggested that deviation from the null was due to a polygenic architecture in which $h_{\text {SNP }}^{2}$ accounted for $\sim 8 \%$ of the population variance in mood instability (observed scale $h_{\mathrm{SNP}}^{2}=0.077(\mathrm{SE} 0.007)$ ), rather than inflation due to unconstrained population structure (LD regression intercept $=0.998($ SE 0.009)).

We observed four independent genomic loci exhibiting genome-wide significant associations with mood instability (Fig. 1, Table 2 and Figs. 3a-d), on chromosome 8 (index SNP rs7829975; CLDN23 and MFHAS1), chromosome 9 (index SNP rs10959826; PTPRD), chromosome 14 (index SNP rs397852991; LTBP2, AREL1, FCF1, YLPM1, PROX2, DLST, RPS6KL1, PGF, EIF2B2 and $M L H 3$ ) and chromosome 18 (index SNP rs8084280; $D C C)$. In total, there were 111 genome-wide significant

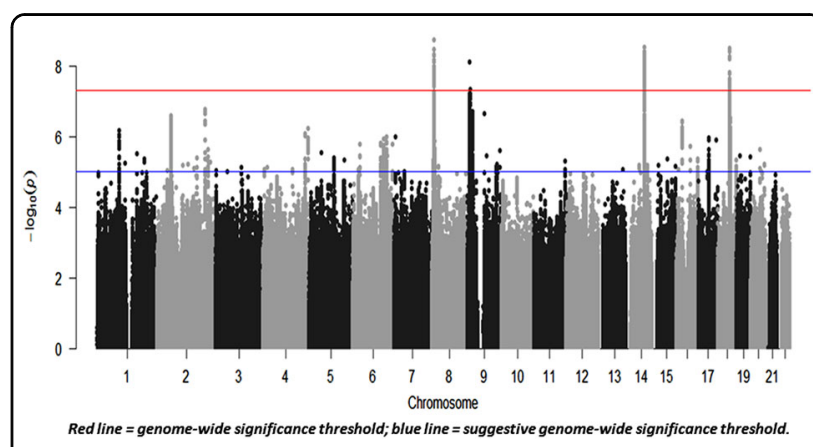

Fig. 1 Manhattan plot of GWAS of mood instability in UK Biobank ( $n=$ 113,968)

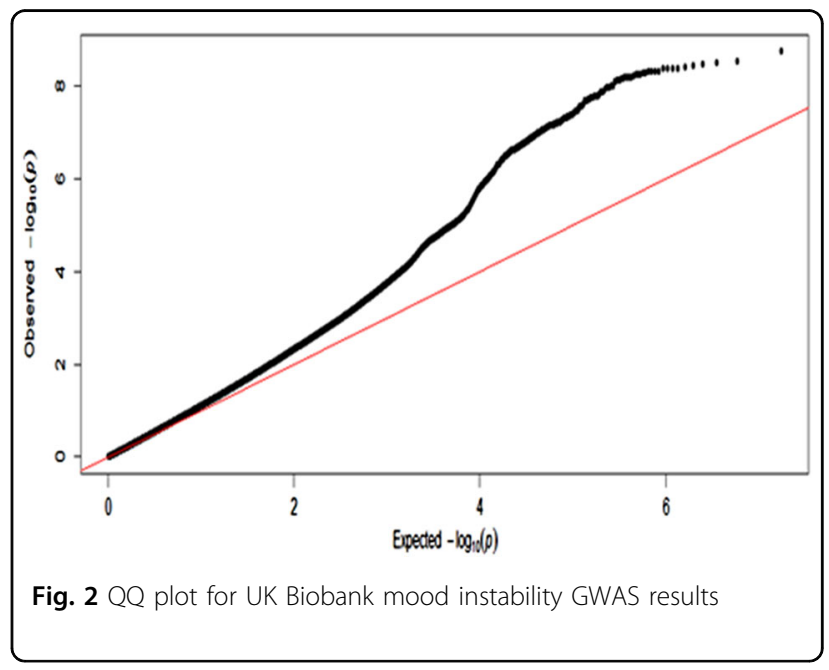




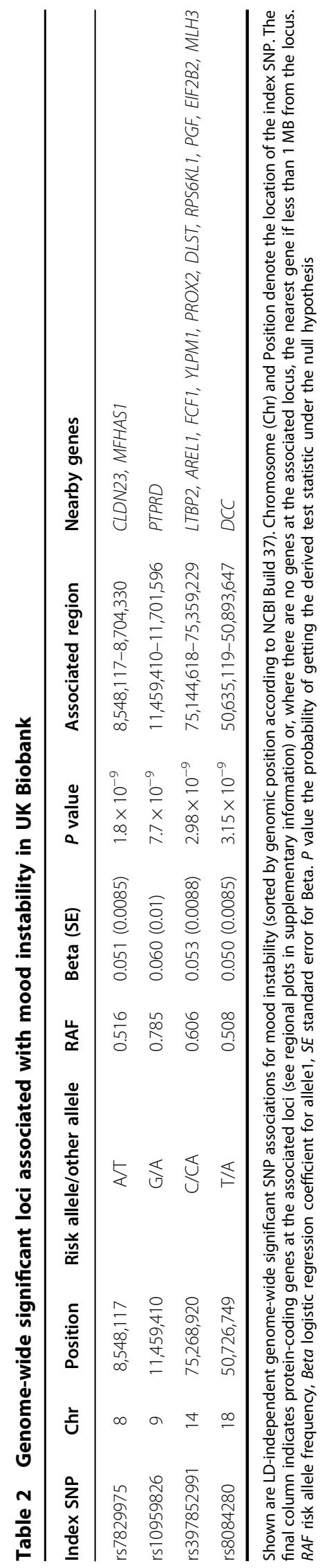

SNPs across all loci. Given the functional alleles that drive association signals in GWAS may not affect the nearest gene, we use the above gene names to provide a guide to location rather than to imply that altered function or expression of those genes are the sources of the association signals.

We also repeated this GWAS for males and females separately (Supplementary Figs. S1 and S2) and for the sample stratified according to median age (age 58 and below, and age 59 and above; Supplementary Figs. S3 and S4). No genome-wide significant loci were observed from these stratified analyses, possibly because of reduced power, apart from the retention of a single genome-wide significant finding at rs8084280 on chromosome 18 (the $D C C$ gene) for males only (Supplementary Fig. S1). There was a high degree of genetic correlation between mood instability in males and females $\left(r_{\mathrm{g}}=1.02, \mathrm{SE}=0.09, p=\right.$ $\left.2.84 \times 10^{-30}\right)$, and between mood instability in the younger and older subgroups $\left(r_{\mathrm{g}}=1.02, \mathrm{SE}=0.09, p=\right.$ $2.67 \times 10^{-27}$ ).

Within supplementary materials, we also present the results of the secondary GWAS of mood instability that was excluded from the case group of 9865 participants with a psychiatric disorder (Supplementary Table S1). This GWAS was used to assess for genetic correlation between mood instability and MDD, BD, schizophrenia, ADHD, anxiety disorders and PTSD, and for the PRS analyses. Supplementary Table S1 shows that the risk allele frequencies (RAFs) of the index SNPs within the four genome-wide significant loci from the primary GWAS were very similar to the RAFs for these same SNPs within this secondary GWAS: for rs7829975 the RAF was 0.516 vs. 0.523 ; for rs 10959826 it was 0.785 vs. 0.789; for rs397852991 it was 0.606 vs. 0.673 ; and for rs8084280 it was 0.508 vs. 0.514). However, it should be noted that, perhaps due to a loss of power from excluding 9865 individuals, only one of these four loci retained genome-wide significance (rs7829975 on chromosome 8).

\section{Genetic correlation of mood instability with MDD, schizophrenia, BD, ADHD, anxiety disorder and PTSD}

We identified strong genetic correlation between mood instability and $\operatorname{MDD}\left(r_{\mathrm{g}}=0.60, \mathrm{SE}=0.07, p=8.95 \times\right.$ $10^{-17}$ ) and a smaller, but significant, correlation between mood instability and both schizophrenia $\left(r_{\mathrm{g}}=0.11, \mathrm{SE}=\right.$ $0.04, p=0.01)$ and anxiety disorders $\left(r_{\mathrm{g}}=0.28, \mathrm{SE}=0.14\right.$, $p=0.04 ;$ Table 3$)$. We did not find significant genetic overlap between mood instability and $\mathrm{BD}\left(r_{\mathrm{g}}=0.01, \mathrm{SE}=\right.$ $0.05, p=0.27)$, ADHD $\left(r_{\mathrm{g}}=0.14, \mathrm{SE}=0.11, p=0.18\right)$ or $\operatorname{PTSD}\left(r_{\mathrm{g}}=0.33, \mathrm{SE}=0.17, p=0.06\right)$. 


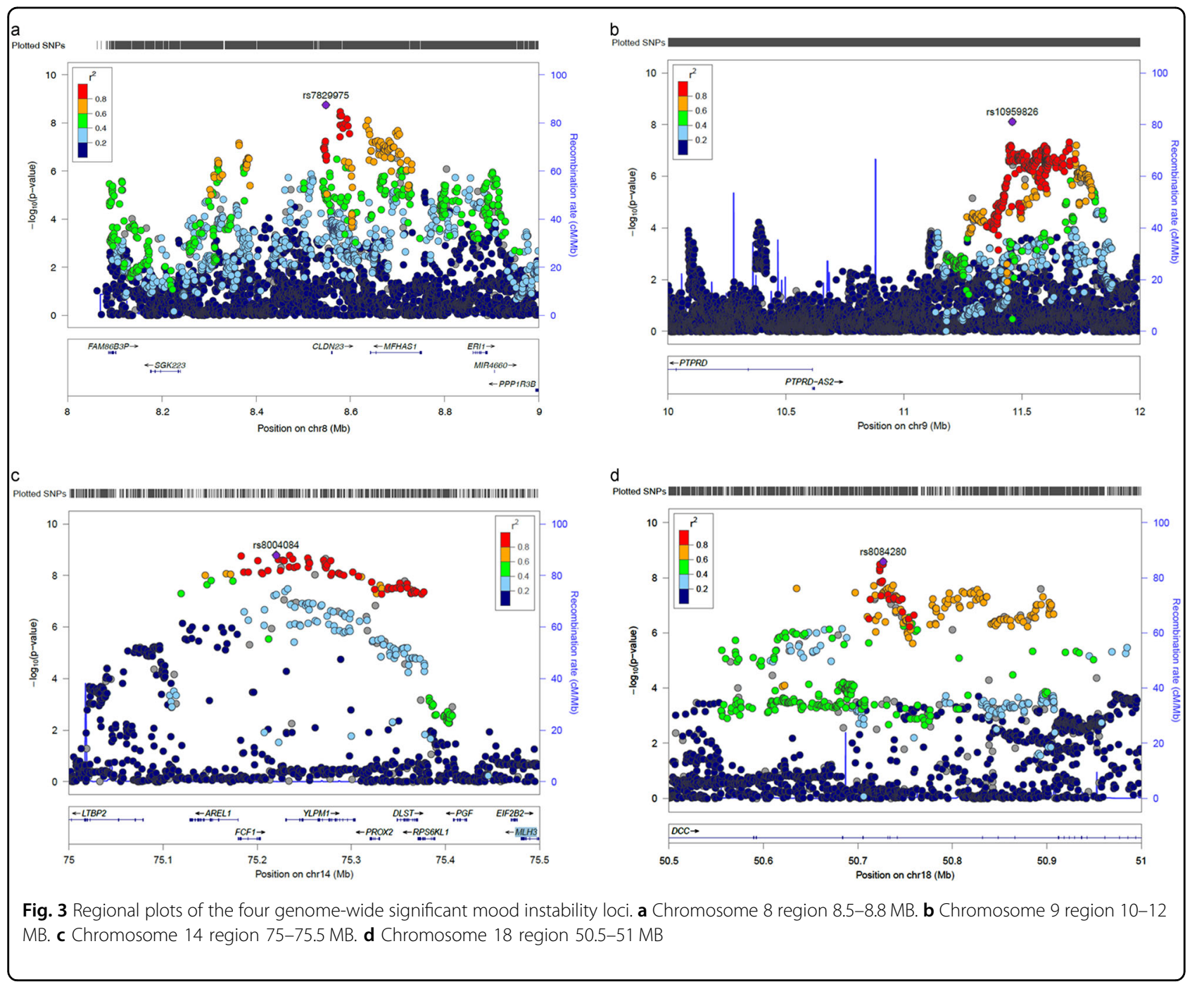

Table 3 Genetic correlation between mood instability and MDD, schizophrenia, BD, PTSD, ADHD and anxiety disorder

\begin{tabular}{lllllllllll}
\hline Phenotype & Rg & se & $\mathbf{z}$ & $\mathbf{p}$ & h2 obs & h2 obs se & h2 int & h2 int se & Gcov int & Gcov int se \\
\hline MDD & 0.6 & 0.07 & 8.32 & $8.95 \times 10^{-17}$ & 0.11 & 0.01 & 0.99 & 0.008 & -0.0019 & 0.006 \\
Schizophrenia & 0.11 & 0.04 & 2.48 & 0.01 & 0.25 & 0.01 & 1.03 & 0.01 & 0.0008 & 0.007 \\
BD & 0.01 & 0.05 & 0.27 & 0.27 & 0.12 & 0.01 & 1.02 & 0.008 & 0.0069 & 0.005 \\
PTSD & 0.33 & 0.17 & 1.9 & 0.06 & 0.10 & 0.004 & 0.99 & 0.007 & 0.0004 & 0.005 \\
ADHD & 0.14 & 0.11 & 1.35 & 0.18 & 0.4 & 0.15 & 1.01 & 0.01 & 0.0046 & 0.004 \\
Anxiety disorder & 0.28 & 0.14 & 2.04 & 0.04 & 0.06 & 0.03 & 1.00 & 0.01 & 0.01 & 0.005 \\
\hline
\end{tabular}

$R_{g}$ genetic correlation with mood instability, SE standard error of the genetic correlation, $Z$ the test statistic, $h^{2}$ obs heritability on the observed scale, $h^{2}$ obs $S E$ the standard error of the heritability, $h^{2}$ int intercept of the heritability, $h^{2}$ int SE standard error of the heritability intercept, Gcov int intercept of the genetic covariance, Gcov int SE standard error of the genetic covariance intercept, MDD major depressive disorder, BD bipolar disorder, PTSD post-traumatic stress disorder, ADHD attention deficit hyperactivity disorder 


\section{PRS analysis of MDD, BD and schizophrenia as predictors of mood instability}

Using the PRS approach, both MDD and schizophrenia had significant positive correlations with mood instability status (for MDD at $p<0.5$ PRS threshold: $\mathrm{OR}=1.029$, 95\% CI $=1.02-1.033, r^{2}=0.023, p=1.00 \times 10^{-34}$ and for schizophrenia at $p<0.1$ PRS threshold: $\mathrm{OR}=1.009,95 \%$ $\mathrm{CI}=1.005-1.014, \quad r^{2}=0.021, \quad p=6.71 \times 10^{-5}$; Supplementary Table S2). There was no evidence of an association between PRS for BD and mood instability. This finding of a positive correlation between PRSs for MDD and schizophrenia and mood instability status (and no such correlation for BD PRS) was consistent across additional analyses stratified for sex and age (Supplementary Tables S3-S6).

\section{Discussion}

We have identified four independent loci associated with mood instability within a large population cohort, in what is to date the only GWAS of this phenotype. We also identified a SNP-based heritability estimate for mood instability of $\sim 8 \%$, and a strong genetic correlation between mood instability and MDD, suggesting substantial genetic overlap between mood instability and vulnerability to MDD. There was also a small but significant genetic correlation between mood instability and schizophrenia and between mood instability and anxiety disorders, but no significant genetic correlation with BD, ADHD or PTSD. PRS analyses found a positive correlation between genes for both MDD and schizophrenia and mood instability status, but this was not the case for BD.

The strong genetic correlation between mood instability and MDD is of interest because it is consistent with the hypothesis that at least part of the pathophysiology of MDD might include a reduced capacity to effectively regulate affective states. In support of this is evidence that individuals with MDD tend to have maladaptive responses to intense emotions, responding with worry, rumination and self-criticism, which can then exacerbate negative emotional states ${ }^{27}$. This maladaptive pattern of responses is also consistent with our finding of a small but significant genetic correlation between mood instability and both anxiety disorder and schizophrenia.

The lack of genetic correlation between mood instability and BD was unexpected, given that mood instability is considered a core deficit in $\mathrm{BD}^{4}$ and was more common in our BD cases than MDD cases. Similarly, a genetic correlation between mood instability, ADHD and PTSD might have been anticipated. This lack of correlation between mood instability and BD/ADHD/PTSD is difficult to account for, but might be explained by the relatively underpowered nature of the BD, ADHD and PTSD GWAS analyses, compared to the analyses used for MDD and schizophrenia. It is worth noting that, although not significant, the magnitude of the genetic correlation between mood instability and ADHD was 0.14. Similarly, the genetic correlation between mood instability and PTSD was not significant but had a magnitude of 0.33 .

It is well documented that MDD occurs more commonly in females than in males, and it is possible that mood instability may be of greater relevance as a crosscutting phenotype for women compared to men. We therefore carried out a GWAS of mood instability for males and females separately (Supplementary Fig. S1 and Fig. S2). These stratified analyses found no genome-wide significant loci for females and only one genome-wide significant locus for males (the previously identified locus on chromosome 18). Furthermore, there was perfect genetic correlation between mood instability in males and females. Although these analyses had reduced power, they suggest that there was no evidence for a large number of sex-specific loci for mood instability. Similarly, we carried out GWAS stratified by age, for those in the sample at or below the median age of 58 and for those above age 58 (Supplementary Figs. S3 and S4). As with stratification by sex, these age-stratified analyses did not identify any genome-wide significant loci, and there was perfect correlation between mood instability in the younger and older subgroups.

It is not possible to be certain which of the genes within associated loci are likely to be most relevant to the pathophysiology of mood instability but several genes of interest were identified. For example, the lead SNP within the associated region on chromosome 18 lies in intron 9 of the DCC netrin 1 receptor (originally named deleted in colorectal cancer; DCC) gene, with no other proteincoding genes for $>500 \mathrm{~kb}$ on either side (Fig. $3 \mathrm{~d}$ ). DCC is the receptor for the guidance cue netrin 1 , which has a central role in the development of the nervous system, including (but not limited to) the organisation and function of mesocorticolimbic dopamine systems ${ }^{28}$. Recent studies have shown a range of human phenotypes associated with loss-of-function mutations in $D C C$, including agenesis of the corpus callosum, learning disabilities and mirror movements, all associated with a large-scale disruption of the development of commissural connectivity and lateralisation ${ }^{29,30}$. Manitt et al. have identified that $D C C$ has a role in regulating the connectivity of the medial prefrontal cortex during adolescence and found that $D C C$ expression was elevated in the brain tissue of antidepressant-free subjects who committed suicide ${ }^{31}$. This suggests a possible role for DCC variants in increasing predisposition to mood instability and mood disorders, as well as related psychopathological phenotypes.

The associated region on chromosome 14 contains at least 10 candidate genes (Table 2 and Fig. 3c). One of these is translation initiation factor $2 B$ subunit beta 
(EIF2B2), mutations in which are known to cause a range of clinically heterogeneous leukodystrophies ${ }^{32}$. Reduced white matter integrity has been consistently associated with negative emotionality traits (such as harm avoidance, neuroticism and trait anxiety) $)^{33}$, as well as with MDD and $\mathrm{BD}^{34}$. It is therefore possible that variation in EIF2B2 may have a role in mood instability.

Another gene within the associated region on chromosome 14 is placental growth factor (PGF), a member of the angiogenic vascular endothelial growth factor (VEGF) family ${ }^{35,36}$, which is expressed at high levels in the placenta and thyroid ${ }^{37}$. PGF has a wide range of functions, including embryonic thyroid development ${ }^{38}$ and immune system function ${ }^{39,40}$, as well as a role in atherosclerosis, angiogenesis in cancer, cutaneous delayed-type hypersensitivity, obesity, rheumatoid arthritis and preeclampsia $^{39,41-44}$. PGF may be of interest because of the long-established association between thyroid dysfunction and both MDD and $\mathrm{BD}^{45}$, along with the recent observation that pre-eclampsia may be a marker for the subsequent development of mood disorders ${ }^{46}$.

Also of interest is the finding that the gene for protein tyrosine phosphatase, receptor type D (PTPRD) lies within $1 \mathrm{Mb}$ of the associated region on chromosome 9 (Fig. 3b). PTPRD encodes a receptor type protein tyrosine phosphatase known to be expressed in the brain and with an organising role at a variety of synapses, including those that play a role in synaptic plasticity ${ }^{47}$. As such, it may have a role in a broad range of psychopathology.

Two of the genomic loci associated with mood instability (on chromosomes eight and nine) overlap with loci found to be associated with neuroticism in a recent GWAS and meta-analysis, which combined data from the UK Biobank cohort, the Generation Scotland cohort and a cohort from the Queensland Institute of Medical Research $^{48}$. The neuroticism study made use of scores on the 12-item EPQ-R-S questionnaire, of which one of the questions was the mood instability question used in the present study. This overlap in findings suggests that mood instability is a key component of neuroticism as defined by the EPQ-R-S and that at least some of the gene variants implicated in mood instability are likely to contribute to the broader phenotype of neuroticism. We did not assess for genetic correlation between mood instability and neuroticism using LDSR because both GWAS outputs were predominantly from the same UK Biobank sample.

\section{Strengths and limitations}

To the best of our knowledge, this is the first reported GWAS of mood instability. It has enabled objective estimates of heritability and genetic correlation with important psychiatric disorders to be made for the first time. In the future, genotyping data for the full UK Biobank sample (502,000 participants) will be available. This increased sample size may identify larger estimates of shared variance between mood instability and psychiatric disorders.

Some important limitations of this work are acknowledged. The mood instability phenotype used was based on response to a single-item question ('Does your mood often goes up and down?'), which may be an imperfect measure of mood instability. Approximately $44 \%$ of the whole UK Biobank cohort answered 'yes' to this question, a much larger proportion than the $13 \%$ of participants classified as having mood instability within the UK APMS ${ }^{2}$. This may be because the assessment of mood instability in the APMS was based on a slightly different question ('Do you have a lot of sudden mood changes') and because respondents had to additionally report that they 'suffered this symptom over the last several years'. Clearly, a potential limitation of self-report is the possibility of responder bias and, further, a more complete and objectively assessed measure of mood instability would have been preferable. However, this was not available to us in the UK Biobank phenotype data set and is unlikely to be feasible to collect within a population cohort of this size.

\section{Conclusions}

Despite a recognition that mood instability is likely to be an important phenotype underpinning a range of psychiatric disorders-particularly mood disorders ${ }^{4}$ - there has to date been very little work on its neural correlates. Early investigations tentatively suggest a role for altered function and/or connectivity of the amygdala ${ }^{49}$, but this is an area that is currently underdeveloped. It is hoped that our findings will stimulate new research on mood instability, which may be a clinically useful and biologically valid trait that cuts across traditional diagnostic categories ${ }^{50}$.

\section{Acknowledgements}

This research was conducted using the UK Biobank resource. UK Biobank was established by the Wellcome Trust, Medical Research Council, Department of Health, Scottish Government and Northwest Regional Development Agency. UK Biobank has also had funding from the Welsh Assembly Government and the British Heart Foundation. Data collection was funded by UK Biobank. J.W. is supported by the JMAS Sim Fellowship for depression research from the Royal College of Physicians of Edinburgh (173558). D.J.S. is supported by an Independent Investigator Award from the Brain and Behaviour Research Foundation (21930) and a Lister Prize Fellowship (173096). A.F. is supported by an MRC Doctoral Training Programme Studentship at the University of Glasgow (MR/K501335/1). The work at Cardiff University was funded by Medical Research Council (MRC) Centre (G0800509) and Programme Grants (G0801418). The funders had no role in the design or analysis of this study, decision to publish or preparation of the manuscript.

\section{Author details}

'Institute of Health and Wellbeing, University of Glasgow, Glasgow, UK. ${ }^{2}$ Department of Medicine Solna, Karolinska Institute, Stockholm, Sweden. ${ }^{3}$ School of Life Sciences, College of Medical, Veterinary and Life Sciences, University of Glasgow, Glasgow, UK. ${ }^{4}$ MRC Centre for Neuropsychiatric Genetics and Genomics, Cardiff University, Cardiff, UK

\section{Competing interests}

The authors declare no competing interests. 
Publisher's note: Springer Nature remains neutral with regard to jurisdictional claims in published maps and institutional affiliations.

\section{Supplementary information}

The online version of this article (doi:10.1038/s41398-017-0012-7) contains supplementary material.

Received: 7 April 2017 Revised: 9 June 2017 Accepted: 9 August 2017 Published online: 30 November 2017

\section{References}

1. Balbuena, L., Bowen, R., Baetz, M. \& Marwaha, S. Mood instability and irritability as core symptoms of major depression: an exploration using rasch analysis. Front. Psychiatry 7, 174 (2016).

2. Marwaha, S., Parsons, N., Flanagan, S. \& Broome, M. The prevalence and clinical associations of mood instability in adults living in England: results from the adult psychiatric morbidity survey 2007. Psychiatr. Res. 205, 262-268 (2013).

3. Cuthbert, B. \& Insel, T. Toward the future of psychiatric diagnosis: the seven pillars of RDoC. BMC Med. 11, 126 (2013).

4. Broome, M. R., Saunders, K. E. A., Harrison, P. J. \& Marwaha, S. Mood instability: significance, definition and measurement. Br. J. Psychiatry 207, 283-285 (2015).

5. Marwaha, S. et al. Affective instability, childhood trauma and major affective disorders. J. Affect. Disord. 190, 764-771 (2016)

6. Howes, O. D. et al. A comprehensive review and model of putative prodromal features of bipolar affective disorder. Psychol. Med. 41, 1567-1577 (2011).

7. Strejilevich, S. A. et al. Mood instability and functional recovery in bipolar disorders. Acta Psychiatr. Scand. 128, 194-202 (2013).

8. Coccaro, E. F., Ong, A. D., Seroczynski, A. D. \& Bergeman, C. S. Affective intensity and lability: heritability in adult male twins. J. Affect. Disord. 136, 1011-1016 (2012).

9. Marwaha, S. et al. How is affective instability defined and measured? A systematic review. Psychol. Med. 44, 1793-1808 (2014).

10. Sudlow, C. et al. UK Biobank: an open access resource for identifying the causes of a wide range of complex diseases of middle and old age. PLoS Med. 12, e1001779 (2015)

11. Eysenck, S. B. G., Eysenck, H. J. \& Barrett, P. A revised version of the psychoticism scale. Pers. Individ. Dif. 6, 21-29 (1985).

12. UK Biobank. Genotype imputation and genetic association studies of UK Biobank, Interim Data Release, 11 September 2015; http://www.ukbiobank.ac. uk/wp-content/uploads/2014/04/imputation_documentation_May2015.pdf.

13. Delaneau, O., Zagury, J.-F. \& Marchini, J. Improved whole-chromosome phasing for disease and population genetic studies. Nat. Method 10, 5-6 (2013).

14. Howie, B., Marchini, J. \& Stephens, M. Genotype imputation with thousands of genomes. G3 1, 457 (2011).

15. Huang, J. et al. Improved imputation of low-frequency and rare variants using the UK10K haplotype reference panel. Nat. Commun. 6, 8111 (2015).

16. UK Biobank. Genotyping of 500,000 UK Biobank participants. Description of sample processing workflow and preparation of DNA for genotyping 11 September 2015; https://biobank.ctsu.ox.ac.uk/crystal/docs/genotyping sample_workflow.pdf.

17. Purcell S. et al. PLINK: A Tool Set for whole-genome association and population-based linkage analyses. Am. J. Hum. Genet. 81, 559-575 (2007).

18. Bulik-Sullivan, B. K. et al. LD Score regression distinguishes confounding from polygenicity in genome-wide association studies. Nat. Genet. 47, 291-295 (2015).

19. Bulik-Sullivan, B. et al. An atlas of genetic correlations across human diseases and traits. Nat. Genet. 47, 1236-1241 (2015).

20. Bipolar Disorder Working Group of the Psychiatric Genomics Consortium Large-scale genome-wide association analysis of bipolar disorder identifies a new susceptibility locus near ODZ4. Nat. Genet. 43, 977-983 (2011).

21. Schizophrenia Working Group of the Psychiatric Genomics Consortium. Biological insights from 108 schizophrenia-associated genetic loci. Nature $\mathbf{5 1 1}$ 421-427 (2014).

22. Major Depressive Disorder Working Group of the Psychiatric Genomics Consortium. A mega-analysis of genome-wide association studies for major depressive disorder. Mol. Psychiatry 18, 497-511 (2013).
23. Neale, B. M. et al. Meta-analysis of genome-wide association studies of attention-deficit/hyperactivity disorder. J Am Acad Child Adolesc Psychiatry 49 884-897 (2010)

24. Otowa, T. et al. Meta-analysis of genome-wide association studies of anxiety disorders. Mol. Psychiatry 21, 1391-1399 (2016).

25. Duncan, L. E. et al. Largest GWAS of PTSD ( $N=20[$ thinsp]070) yields genetic overlap with schizophrenia and sex differences in heritability. Mol. Psychiatry (2017).

26. Smith, D. J. et al. Prevalence and characteristics of probable major depression and bipolar disorder within UK Biobank: cross-sectional study of 172,751 participants. PLOS ONE 8, e75362 (2013).

27. Mennin, D. S. \& Fresco, D. M. What, me worry and ruminate about DSM-5 and RDoC? The importance of targeting negative self-referential processing. Clin. Psychol. 20, 258-267 (2013).

28. Manitt, C. et al. The Netrin receptor DCC is required in the pubertal organization of mesocortical dopamine circuitry. J. Neurosci. 31, 8381-8394 (2011).

29. Jamuar, S. S. et al. Biallelic mutations in human DCC cause developmental split-brain syndrome. Nat. Genet. 49, 606-612 (2017).

30. Marsh, A. P. L. et al. Mutations in DCC cause isolated agenesis of the corpus callosum with incomplete penetrance. Nat. Genet. 49, 511-514 (2017).

31. Manitt, C. et al. dcc orchestrates the development of the prefrontal cortex during adolescence and is altered in psychiatric patients. Transl. Psychiatry $\mathbf{3}$ e338 (2013)

32. Horzinski, L. et al. Eukaryotic initiation factor 2B (elF2B) GEF activity as a diagnostic tool for EIF2B-related disorders. PLOS ONE 4, e8318 (2009).

33. Mincic, A. M. Neuroanatomical correlates of negative emotionality-related traits: a systematic review and meta-analysis. Neuropsychologia 77, 97-118 (2015).

34. Sexton, C. E., Mackay, C. E. \& Ebmeier, K. P. A systematic review of diffusion tensor imaging studies in affective disorders. Biol. Psychiatry 66, 814-823 (2009).

35. De Falco, S. The discovery of placenta growth factor and its biological activity. Exp. Mol. Med. 44, 1-9 (2012).

36. Xie, T. et al. VEGF-related polymorphisms identified by GWAS and risk for major depression. Transl. Psychiatry 7, e1055 (2017).

37. Viglietto, G. et al. Upregulation of vascular endothelial growth factor (VEGF and downregulation of placenta growth factor (PIGF) associated with malignancy in human thyroid tumors and cell lines. Oncogene 11, 1569-1579 (1995).

38. Korevaar, T. I. et al. Soluble Flt1 and placental growth factor are nove determinants of newborn thyroid (dys)function: the generation R study. J. Clin. Endocrinol. Metab. 99, E1627-E1634 (2014).

39. Oura, $\mathrm{H}$. et al. A critical role of placental growth factor in the induction of inflammation and edema formation. Blood 101, 560-567 (2003).

40. Luttun, A. et al. Revascularization of ischemic tissues by PIGF treatment, and inhibition of tumor angiogenesis, arthritis and atherosclerosis by anti-Flt1. Nat Med. 8, 831-840 (2002).

41. Carmeliet, P. et al. Synergism between vascular endothelial growth factor and placental growth factor contributes to angiogenesis and plasma extravasation in pathological conditions. Nat. Med. 7, 575-583 (2001).

42. Yoo, S. A. et al. Role of placenta growth factor and its receptor flt-1 in rheumatoid inflammation: a link between angiogenesis and inflammation. Arthritis Rheum. 60, 345-354 (2009).

43. Lijnen, H. R. et al. Impaired adipose tissue development in mice with inactivation of placental growth factor function. Diabetes 55, 2698-2704 (2006).

44. Chappell, L. C. et al. Diagnostic accuracy of placental growth factor in women with suspected preeclampsia: a prospective multicenter study. Circulation $\mathbf{1 2 8}$, 2121-2131 (2013).

45. Bauer, M., Goetz, T., Glenn, T. \& Whybrow, P. C. The thyroid-brain interaction in thyroid disorders and mood disorders. J. Neuroendocrinol. 20, 1101-1114 (2008).

46. Bergink, V. et al. Pre-eclampsia and first-onset postpartum psychiatric episodes: a Danish population-based cohort study. Psychol. Med. 45, 3481-3489 (2015).

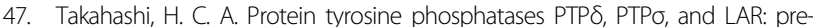
synaptic hubs for synapse organization. Trends Neurosci. 36, 522-534 (2013).

48. Smith, D. J. et al. Genome-wide analysis of over 106,000 individuals identifies 9 neuroticism-associated loci. Mol. Psychiatry 21, 749-757 (2016)

49. Broome, M. R., He, Z, Iftikhar, M. Eyden, J. \& Marwaha, S. Neurobiological and behavioural studies of affective instability in clinical populations: a systematic review. Neurosci. Biobehav. Rev. 51, 243-254 (2015).

50. Insel, $\mathrm{T}$. The $\mathrm{NIMH}$ research domain criteria (RDoC) project: precision medicine for psychiatry. Am. J. Psychiatry 171, 395-397 (2014). 